\title{
Percutaneous Spinal Ablation in a Sheep Model: Protective Capacity of an Intact Cortex, Correlation of Ablation Parameters with Ablation Zone Size, and Correlation of Postablation MRI and Pathologic Findings
}

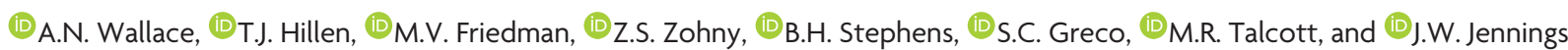

\begin{abstract}
BACKGROUND AND PURPOSE: Despite the growing use of percutaneous ablation therapy for the treatment of metastatic spine disease, several issues have yet to be fully addressed. Our aims were to determine whether the vertebral body cortex protects against ablationinduced spinal cord injury; correlate radiofrequency, cryo-, and microwave ablation parameters with resulting spinal ablation zone dimensions and describe normal spinal marrow postablation changes on MR imaging.
\end{abstract}

MATERIALS AND METHODS: Ten thoracolumbar vertebrae in 3 sheep were treated with radiofrequency ablation, cryoablation, or microwave ablation under fluoroscopic guidance. Technique parameters were chosen to produce ablation zones that exceeded the volume of the vertebral bodies in sheep 1 and were confined to the vertebrae in sheep 2 and 3 . Expected ablation zone dimensions were based on data provided by the device manufacturers. Postablation MR imaging was performed at 48 hours (sheep 1) or 7 days (sheep 2 and 3$)$.

RESULTS: In sheep 1, cryoablation and microwave ablations extended into the spinal canal and caused histologically confirmed neurologic injury, but radiofrequency ablation did not. The mean difference between the lengths of the radiofrequency ablation zone dimensions measured on gross pathology compared with those expected was $9.6 \pm 4.1 \mathrm{~mm}$. The gross pathologic cryo- and microwave ablation zone dimensions were within $1 \mathrm{~mm}$ of those expected. All modalities produced a nonenhancing ablation zone with a rim of enhancement, corresponding histologically to marrow necrosis and hemorrhagic congestion.

CONCLUSIONS: An intact cortex appears to protect against radiofrequency ablation-induced spinal cord injury, but not against nonimpedance-based modalities. Ablation dimensions produced by microwave and cryoablation are similar to those expected, while radiofrequency ablation dimensions are smaller. Ablation of normal marrow produces a rim of enhancement at the margin of the ablation zone on MR imaging.

ABBREVIATIONS: MWA = microwave ablation; RFA = radiofrequency ablation

$\mathbf{P}$ ercutaneous, image-guided ablation is a minimally invasive therapy for metastatic spine disease. Several ablation modalities are commercially available, including radiofrequency ablation (RFA), cryoablation, and microwave ablation (MWA). RFA and MWA heat the tumor to temperatures above $60^{\circ} \mathrm{C}$, at which

\footnotetext{
Received December 28, 2016; accepted after revision March 17, 2017.

From the Mallinckrodt Institute of Radiology (A.N.W., T.J.H., M.V.F., J.W.J.), Department of Neurosurgery (Z.S.Z., B.H.S.), and Division of Comparative Medicine (S.C.G., M.R.T.), Washington University, St Louis, Missouri.

The authors received ablation probes from DFINE, Galil Medical, and Medtronic for this study.

Please address correspondence to Adam N. Wallace, MD, Mallinckrodt Institute of Radiology, Washington University, 510 South Kingshighway Blvd, St Louis, MO 63141; e-mail: wallacea@mir.wustl.edu

http://dx.doi.org/10.3174/ajnr.A5228
}

point protein denaturation, immediate cell death, and coagulative necrosis occur. ${ }^{1}$ Mechanisms of cryoablation-induced cell death include osmotic bursting, intracellular ice formation, coagulative necrosis, free radical release and apoptosis in response to biochemical stress, and stimulated immunologic targeting of tumor cells. $^{2}$ For patients with painful spinal metastases who do not respond or have a contraindication to radiation therapy, ablation may produce clinically significant pain palliation. ${ }^{3-7}$ Additionally, Wallace et $\mathrm{al}^{8}$ recently reported a 1-year radiologic local control rate of 70\% (21/30) after RFA of spinal metastases, and Tomasian et $\mathrm{al}^{5}$ achieved a $97 \%$ (30/31) radiologic local control rate of spinal metastases treated with cryoablation after a median follow-up of 10 months (range, 1-24 months). As a result, recent treatment algorithms for metastatic spine disease have incorporated ablation as an alternative option for pain palliation and local tumor control. ${ }^{9}$

AJNR Am J Neuroradiol 38:1653-59 Aug 2017 www.ajnr.org

1653 


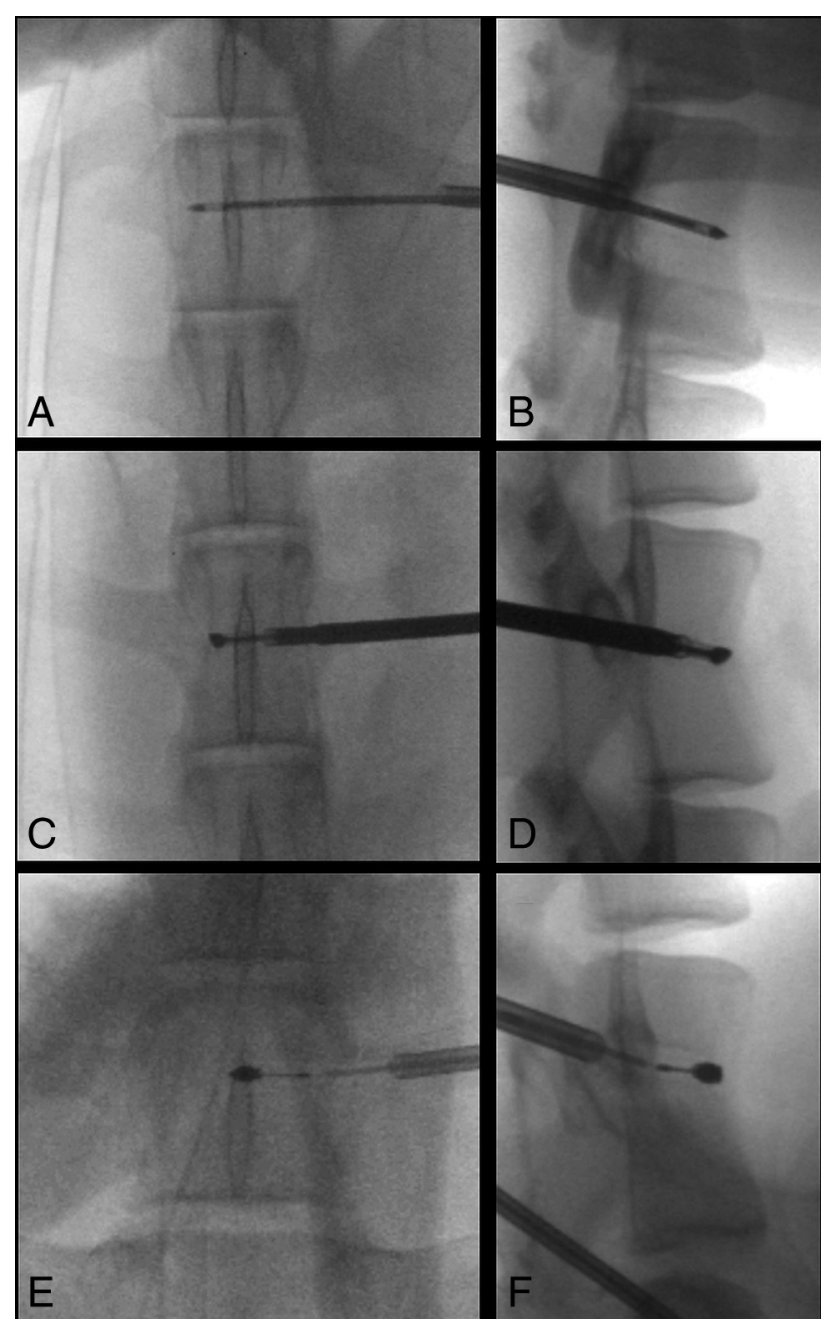

FIG 1. Fluoroscopic documentation of ablation probe placement in sheep. Anteroposterior $(A, C$, and $E)$ and lateral $(B, D$, and $F)$ fluoroscopic images show transpedicular placement of the cryoablation probe within the T14 vertebra $(A$ and $B)$, the radiofrequency ablation probe within the $L 3$ vertebra $(C$ and $D)$, and the microwave ablation probe within the $L 6$ vertebra ( $E$ and $F)$.

Table 1: Technical parameters used for radiofrequency ablation, expected minor-axis diameter of the ablation zones based on nonanimal models, and measured ablation zone diameters on MRI and gross pathology

\begin{tabular}{|c|c|c|c|c|c|c|}
\hline \multirow[b]{2}{*}{ Watts } & \multicolumn{2}{|c|}{$\begin{array}{l}\text { Thermocouple } \\
\text { Temperatures }\left({ }^{\circ} \mathrm{C}\right) \\
\end{array}$} & \multirow{2}{*}{$\begin{array}{c}\text { Ablation } \\
\text { Time } \\
\text { (sec) }\end{array}$} & \multicolumn{3}{|c|}{$\begin{array}{c}\text { Diameter of Ablation Zone Orthogonal } \\
\text { to Ablation Probe (mm) }\end{array}$} \\
\hline & Proximal & Distal & & Expected & MRI & Gross Pathology \\
\hline 10 & 72 & 51 & 92 & 20.0 & 13.4 & 13.8 \\
\hline 5 & 58 & 42 & 107 & 16.6 & 9.2 & 10.0 \\
\hline 5 & 51 & 40 & 42 & 13.0 & 7.3 & 8.0 \\
\hline
\end{tabular}

Table 2: Technical parameters used for cryoablation, expected minor-axis diameter of the ablation zones based on nonanimal models, and measured ablation zone diameters on MRI and gross pathology

\begin{tabular}{lcccccc}
\hline & & $\begin{array}{c}\text { Freeze } \\
\text { Intensity } \\
\text { Pycles }\end{array}$ & $\begin{array}{c}\text { Thaw } \\
\text { Probe }\end{array}$ & $\begin{array}{c}\text { (min) } \\
(\mathbf{m i n})\end{array}$ & $\begin{array}{c}\text { Diameter of Ablation Zone Orthogonal } \\
\text { to Ablation Probe (mm) }\end{array}$ \\
\hline IceSphere & 60 & 6.0 & 7.0 & 20.0 & 19.2 & 20.1 \\
IceSeed & 50 & 4.0 & 5.0 & 6.0 & 6.6 & 7.4 \\
IceSeed & 50 & 3.5 & 5.0 & 6.0 & 6.4 & 7.0 \\
IceSeed & 50 & 3.0 & 5.0 & 5.0 & 5.5 & 6.8 \\
\hline
\end{tabular}

Despite the growing use of percutaneous ablation therapy for the treatment of metastatic spine disease, several important issues have yet to be fully addressed. First, most spinal metastases involve the posterior vertebral body where complete tumor ablation is essential to prevent localized epidural invasion and spinal cord compression. ${ }^{10,11}$ However, it is unclear whether intact cortex acts as a protective barrier for the spinal cord and exiting nerve roots during ablation of the posterior vertebral body. ${ }^{12,13}$ Second, adequate tumor ablation requires accurate correlations between technique parameters and the resulting ablation zone dimensions. However, existing correlations derived from the manufacturers' preclinical data are based on soft-tissue and artificial models that have different thermal and electrical conductive properties to bone. ${ }^{14}$ Last, although MR imaging is typically used to assess for local tumor control after ablation of spinal metastases, ${ }^{15}$ diagnosis of residual tumor is limited by a paucity of literature describing ablation-induced MR imaging findings in normal spinal marrow.

The purposes of this study were to determine whether intact posterior vertebral body cortex protects the spinal cord against radiofrequency-, cryo-, or microwave ablation-induced injury, to correlate the technical parameters of each ablation technique with the resulting dimensions of the ablation zone, and to describe normal spinal marrow postablation changes on MR imaging.

\section{MATERIALS AND METHODS}

The protocol of this study was approved by our institutional animal care and use committee.

\section{Ablation Technique}

Ablations were performed under fluoroscopic guidance by 4 radiologists (A.N.W., T.J.H., M.V.F., J.W.J.) with 1-9 years of experience performing musculoskeletal tumor ablation. Ten vertebral bodies in 3 healthy living adult female sheep (weight range, $55-60 \mathrm{~kg}$ ) were ablated. All 3 sheep were sedated by a veterinarian staff member with an intramuscular injection of ketamine hydrochloride (2-10 $\mathrm{mg} / \mathrm{kg})$ and xylazine $(0.2 \mathrm{mg} / \mathrm{kg})$, intubated, and maintained under inhaled isoflurane $(1 \%-5 \%)$ for general anesthesia. Mechanical ventilation was used throughout the procedure. The target vertebrae were localized by using a single-plane fluoroscopy unit, and the planned skin entry sites were sterilized with betadine scrubs and draped. Fiveto $10-\mathrm{mm}$ incisions were made at each skin entry site with a No. 11 blade to accommodate the percutaneous instruments. Unilateral transpedicular osseous channels into the vertebral bodies were created for ablation probe placement by using the OnControl system (Vidacare, Shavano Park, Texas), which consists of a 10-ga outer can- 
Table 3: Technical parameters used for microwave ablation, expected minor-axis diameter of the ablation zones based on nonanimal models, and measured ablation zone diameters on MRI and gross pathology

\begin{tabular}{lcccc}
\hline & & \multicolumn{2}{c}{$\begin{array}{c}\text { Diameter of Ablation Zone } \\
\text { Obthogonal to Ablation Probe (mm) }\end{array}$} \\
\cline { 3 - 5 } Watts & $\begin{array}{c}\text { Ablation } \\
\text { Time }\end{array}$ & Mected & MRI & $\begin{array}{c}\text { Gross } \\
\text { Pathology }\end{array}$ \\
\hline 100 & 50 & 20.0 & 17.4 & 20.0 \\
100 & 15 & 9.5 & 7.9 & 9.0 \\
100 & 10 & 6.0 & 5.9 & 6.5 \\
\hline
\end{tabular}

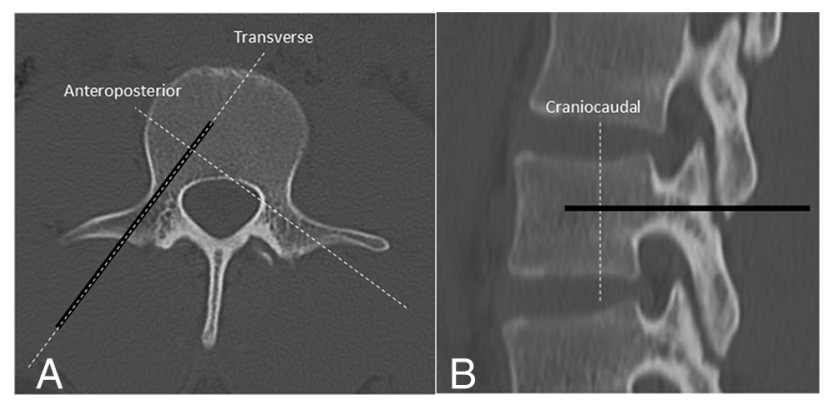

FIG 2. Anteroposterior $(A)$ and lateral $(B) C T$ images of a normal lumbar vertebra. The thick black line represents the unilateral transpedicular trajectory of the ablation probe. By definition, the transverse and anteroposterior dimensions of the ablation zone are parallel and perpendicular to the ablation probe in the axial plane, respectively, and the craniocaudal dimension of the ablation zone is perpendicular to the ablation probe in the sagittal plane.

nula and 12-ga inner osteotome. The locations of each probe were documented fluoroscopically (Fig 1).

\section{Ablation Parameters}

The technical parameters and expected dimensions of each ablation zone based on preclinical data provided by the manufacturers are detailed in Tables 1-3. In sheep 1, cryoablation, RFA, and MWA were performed in the T14, L3, and L6 vertebral bodies, respectively. Sheep most commonly have 14 rib-bearing thoracic and 6 lumbar vertebrae, and the spinal cord terminates caudal to the sacrum. Each ablation probe tip was placed $5 \mathrm{~mm}$ from the posterior vertebral body cortex on a true lateral fluoroscopic projection. The parameters of radiofrequency, cryo-, and microwave ablation were chosen to produce ablation zones with anteroposterior, transverse, and craniocaudal dimensions of $30 \times 20 \times 20$ $\mathrm{mm}, 25 \times 20 \times 20 \mathrm{~mm}$, and $20 \times 20 \times 20 \mathrm{~mm}$, respectively. The orientation of each dimension of the ablation zone with respect to the ablation probe was defined as depicted in Fig 2. Thus, for each ablation, the radius of the ablation zone extending from the probe tip toward the posterior vertebral body cortex was expected to be $10 \mathrm{~mm}$. Every third vertebra was treated to ensure correct localization of histologic ablation-induced spinal cord injury.

In sheep 2 and 3, each ablation probe was placed in the center of the vertebral body, and the parameters of each ablation were chosen to produce an ablation volume that would be confined to the vertebra on the basis of preclinical data provided by the manufacturers. In sheep 2, RFA was performed at T14; MWA, at L3; and cryoablation, at L6. In sheep 3, RFA was performed at T14; MWA, at L2; and cryoablation, at L4 and L6.

RFAs were performed with the STAR Tumor Ablation System
(DFINE, San Jose, California). Ablations can be performed at power settings of 5 or $10 \mathrm{~W}$. Two thermocouples located on the shaft of the probe enable real-time monitoring of temperatures 10 and $15 \mathrm{~mm}$ from the center of the ablation zone, and the dimensions of the ablation zone are estimated from these maximum temperature readings. The expected diameter of each RFA zone orthogonal to the probe is based on the manufacturer's thermal distribution curves derived from ex vivo porcine liver models.

Cryoablations were performed with the Visual-ICE Cryoablation System (Galil Medical, Arden Hills, Minnesota) with either the IceSeed 1.5 or IceSphere 1.5 cryoprobe. For a given cryoprobe, the volume of the ablation zone is controlled by changing the intensity (percentage maximum) and the duration of the freeze cycle. In the present study, 2 freeze cycles were performed and the probe was actively heated to thaw the tissue between cycles. The expected diameter of each ablation zone orthogonal to the cryoprobe was based on the $-40^{\circ} \mathrm{C}$ isotherm dimensions produced in a $37^{\circ} \mathrm{C}$ gel bath.

MWAs were performed by using the Emprint Ablation System (Medtronic, Minneapolis, Minnesota). Ablations were performed at a power setting of $100 \mathrm{~W}$ for 10,15 , and 50 seconds. Power settings of 45 and $75 \mathrm{~W}$ are also available. The expected diameter of each MWA zone was based on an ex vivo porcine kidney model.

\section{Postablation Evaluation}

The sheep were clinically evaluated by a veterinarian immediately after each procedure for signs of pain or ablation-induced nerve injury, including lameness or abnormal movement. Subcutaneous buprenorphine, $0.005-0.01 \mathrm{mg} / \mathrm{kg}$, was administered every 10-12 hours, and carprofen, $2-4 \mathrm{mg} / \mathrm{kg}$, was administered every 12-24 hours for 2 days postoperatively. The sheep were then periodically monitored by veterinary staff before sacrifice for evidence of procedure-related complications.

\section{MR Imaging}

In sheep 1, MR imaging was performed 48 hours after treatment owing to clinical signs of spinal cord injury and the ethical necessity for euthanasia earlier than the planned 7-day time point. MR imaging was performed 7 days after treatment of sheep 2 and 3. Images were obtained on a Magnetom Trio 3T scanner (Siemens, Erlangen, Germany) using the spine coil within the table of the scanner. The protocol included T1-weighted conventional spinecho images (TR, $783 \mathrm{~ms}$; TE, $10 \mathrm{~ms}$ ), T2-weighted fast spin-echo images with fat suppression (TR, $3500 \mathrm{~ms}$; TE, $94 \mathrm{~ms}$ ), and postcontrast conventional spin-echo T1-weighted images with fat suppression obtained after the intravenous bolus administration of $0.1 \mathrm{mmol} / \mathrm{kg}$ of gadoversetamide (Optimark; Covidien, Irvine, California). All images were acquired with a $2.5-\mathrm{mm}$ section

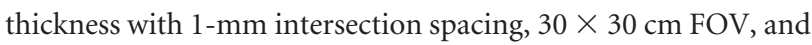
$288 \times 384$ matrix. On MR imaging, the ablation zone was defined as the volume of nonenhancement on contrast-enhanced T1weighted images with fat suppression. ${ }^{15}$ The measurements of MR imaging ablation zones were performed on high-resolution MR imaging workstation monitors. The anteroposterior, transverse, and craniocaudal ablation zone dimensions were measured for sheep 2 and 3. Only the transverse and craniocaudal dimen- 
sions of each ablation zone were measured in sheep 1, because the anteroposterior dimensions was not confined to the vertebral body marrow. MR signal and enhancement characteristics of the ablation zones of each technique were also subjectively reviewed by the first and senior authors. No interpretations were discrepant.

\section{Pathology}

Following MR imaging, the sheep were euthanized by barbiturate overdose (intravenous pentobarbital, $150 \mathrm{mg} / \mathrm{kg}$ ) and the vertebral bodies and spinal cord were harvested by senior neurosurgery residents (B.H.S., Z.S.Z.). Tissues were fixed in $10 \%$ neutral buffered formalin. After fixation, the vertebral bodies were decalcified in hydrochloric acid-ethylenediaminetetraacetic acid decalcifier (EKI, Joliet, Illinois) to permit cross-sectioning of the bones for gross examination of the ablation sites and trimming of tissues for histopathology. The diameters of the pale tan necrosis orthogonal probe tract were measured once by the first author and a veteri-

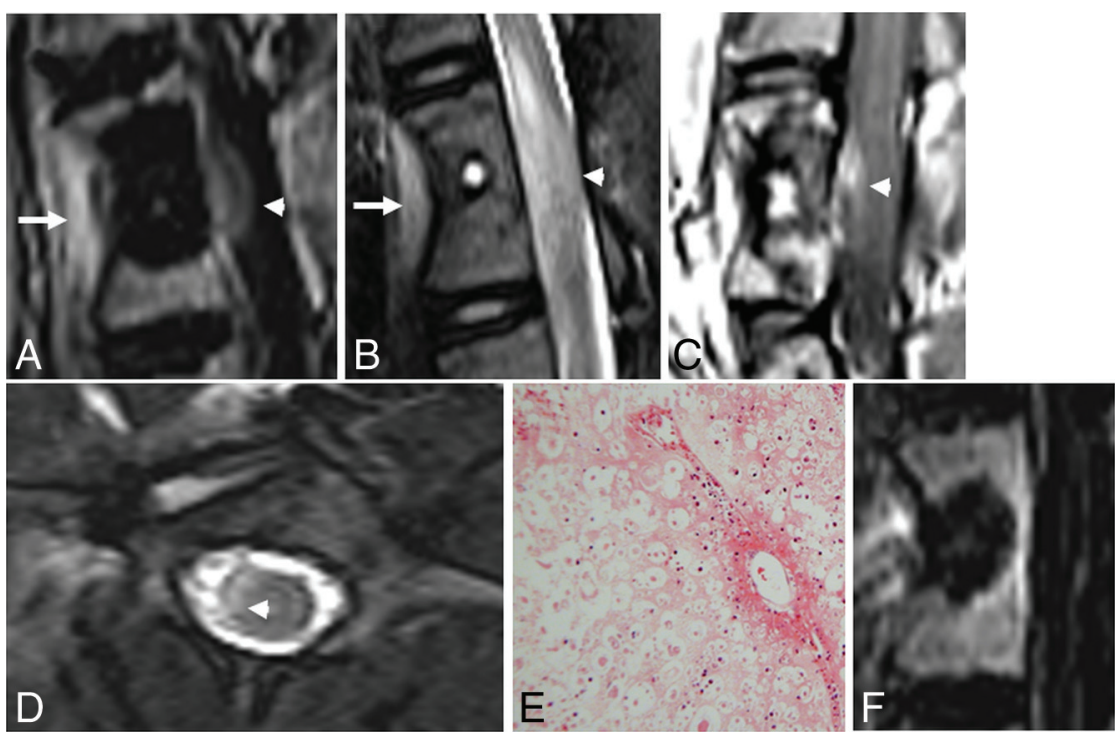

FIG 3. Sagittal postcontrast T7-weighted image with fat suppression $(A)$ and a T2-weighted image $(B)$ of the vertebral level treated with cryoablation show the ablation zone extending beyond the posterior vertebral body into the spinal canal. There is enhancement and intramedullary T2 signal hyperintensity in the ventral aspect of the edematous spinal cord (white arrowheads). There is also inflammation in the soft tissues ventral to the spine where the ablation zone extended beyond the anterior vertebral body cortex (white arrows). Sagittal postcontrast T1-weighted image $(C)$ and axial T2-weighted image $(D)$ of the vertebral level treated with microwave ablation similarly show extension of the ablation zone into the spinal canal (white arrowheads). E, Hematoxylin-eosin staining of the spinal cord at the level of the cryoablated vertebra at $\times 100$ total magnification shows axonal necrosis and edema. Similar findings were seen at the microwaveablated level (not shown). F, Sagittal postcontrast T1-weighted image with fat suppression of the radiofrequency-ablated vertebra shows the posterior margin of the ablation zone confined to the vertebral body.

Table 4: Mean differences among the anteroposterior, transverse, and craniocaudal ablation zone dimensions expected on the basis of manufacturers' preclinical data and those measured on MRI and gross pathology ${ }^{\mathrm{a}}$

\begin{tabular}{|c|c|c|c|c|c|c|c|}
\hline & \multicolumn{3}{|c|}{ Expected vs MRI (mm) } & \multicolumn{2}{|c|}{$\begin{array}{c}\text { Expected vs } \\
\text { Pathology (mm) }\end{array}$} & \multicolumn{2}{|c|}{ MRI vs Pathology (mm) } \\
\hline & AP & TV & $\mathrm{CC}$ & TV & $\mathrm{CC}$ & TV & $\mathrm{CC}$ \\
\hline Radiofrequency ablation & $6.7 \pm 0.9$ & $11.0 \pm 2.5$ & $6.6 \pm 0.7$ & $13.4 \pm 2.1$ & $5.9 \pm 0.7$ & $-1.2 \pm 0.7$ & $-0.8 \pm 0.1$ \\
\hline Cryoablation & $-0.5 \pm 0.3$ & $0.1 \pm 0.7$ & $-0.1 \pm 0.7$ & $-0.4 \pm 0.6$ & $-1.0 \pm 0.5$ & $-3.4 \pm 0.2$ & $-0.9 \pm 0.3$ \\
\hline Microwave ablation & $0.9 \pm 0.4$ & $0.3 \pm 0.5$ & $1.4 \pm 1.0$ & $0.1 \pm 0.5$ & $0 \pm 0.4$ & $-0.8 \pm 0.2$ & $-0.9 \pm 0.3$ \\
\hline
\end{tabular}

Note:-AP indicates anteroposterior; TV, transverse; CC, craniocaudal.

a A positive value indicates that the value listed first in the upper row was larger than the value listed second. For example, a positive value under the "Expected vs MRI" column indicates that the expected ablation zone dimension was larger than that measured on MRI. Orientations of the ablation zone dimensions with respect to the ablation probe are defined in Fig 2. nary pathologist with a Westcott 6" Clear Plastic Acrylic Ruler (Acme United Corporation, Fairfield, Connecticut). Because the vertebral bodies could be sectioned in only 1 plane, only the transverse and craniocaudal diameters of the ablation zones could be measured. Tissues were then paraffin-embedded, sectioned at 5 $\mu \mathrm{m}$, and stained with hematoxylin-eosin for histopathologic RESULTS

Immediately after recovering from anesthesia, sheep 1 demonstrated bilateral lower extremity weakness, which did not improve during 48 hours of observation. MR imaging showed intramedspinal cord at the level of the cryoablated vertebra consistent with ablation-induced injury. Similar findings were seen along the ventrolateral aspect of the spinal cord at the level of the microwave-ablated vertebra, likely due to a slightly more oblique tologically, the ablation zone was characterized by white matter necrosis with cavitation and axonal swelling and degeneration (Fig 3). The RFA zone measured $13.8 \mathrm{~mm}$ in the craniocaudal dimension. Because the RFA probe was placed only $5 \mathrm{~mm}$ from the posterior vertebral body cortex, the RFA zone should have extended into the spinal canal. Yet, histologic evaluation of the spinal cord at this level showed no evidence of ablation-induced injury.

Tables 1-3 summarize the correlations between the technical parameters of each technique and the resulting dimensions of the gross pathologic ablation zones. The mean differences in millimeters among the anteroposterior, transverse, and craniocaudal ablation zone dimensions expected on the basis of the manufacturers' preclinical data and those measured on MR imaging and gross pathology are presented in Table 4. The dimensions of the RFA zones measured on gross pathology were smaller than those expected on the basis of correlations provided by the manufacturer. The mean differences between the lengths of the transverse and craniocauullary T2 hyperintensity and enhancement involving the ventral 
Table 5: MRI, gross, and histopathologic findings produced by all 3 ablation modalities

\begin{tabular}{lllll}
\hline & \multicolumn{1}{c}{ TIWI } & \multicolumn{1}{c}{ T2WI } & \multicolumn{1}{c}{ CE-TIWI } & \multicolumn{1}{c}{ Histopathology } \\
\hline Probe track & Hyperintense & Hyperintense & Enhancement & $\begin{array}{l}\text { Hemorrhage, trabecular fragments, serum exudate, } \\
\text { granulation tissue }\end{array}$ \\
$\begin{array}{llll}\text { Center } & \text { Slightly hyperintense } & \text { Slightly hypointense } & \text { No enhancement } \\
\text { Rim } & \text { Hyperintense } & \text { Hyperintense } & \text { Enhancement }\end{array}$ & $\begin{array}{l}\text { Marrow necrosis, granulation tissue, intact trabeculae } \\
\text { Hemorrhagic congestion }\end{array}$ \\
\hline
\end{tabular}

Note:-CE indicates contrast-enhanced.
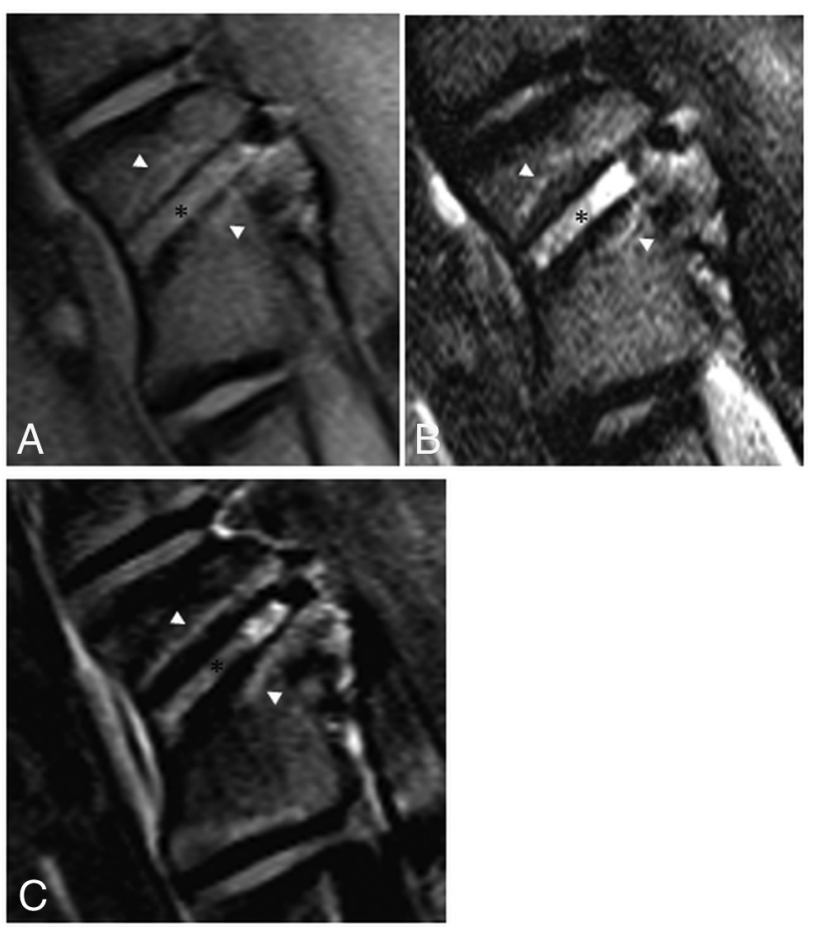

FIG 4. MR imaging findings 7 days after RFA of an $L 2$ vertebral body in a sheep model. A, T7-weighted oblique sagittal MR imaging (left, anterior; right, posterior) shows $\mathrm{Tl}$ hyperintense soft tissue within the probe tract (black asterisk) outlined by a signal void. The surrounding ablation zone is isointense to normal marrow and outlined by a thin hyperintense rim (white arrowheads). B, T2-weighted oblique sagittal MR imaging with fat suppression shows the hyperintense probe tract (black asterisk) and slightly hypointense ablation zone surrounded by a hyperintense rim (white arrowheads). C, T1-weighted, postcontrast subtraction images show enhancement along the probe tract (black asterisk) and the nonenhancing ablation zone surrounded by a rim of enhancement (white arrowheads).

dal RFA zone dimensions measured on gross pathology compared with those expected were $13.4 \pm 2.1$ and $5.9 \pm 0.7 \mathrm{~mm}$, respectively. In contrast, the cryo- and microwave ablation zone dimensions measured on gross pathology were within $1 \mathrm{~mm}$ of those expected.

All ablation modalities produced the same subjective signal and enhancement characteristics on MR imaging (Table 5). Relative to normal marrow, the probe tracts demonstrated $\mathrm{T} 1$ isointense, T2 hyperintense, and enhancing tissue outlined by signal void. The ablation zones were defined by nonenhancing tissue that was slightly $\mathrm{T} 1$ hyperintense and $\mathrm{T} 2$ isointense and delineated by a thin rim of $\mathrm{T} 1$ and $\mathrm{T} 2$ hyperintensity and enhancement (Fig 4). The gross and histologic findings seen with all modalities were qualitatively similar (Table 5). Gross examination revealed discrete pale tan ablation zones. Histologically, the ablation zones were characterized by widespread marrow necrosis with areas of granulation tissue and edematous stroma.
Surrounding the ablation zones were rims of hemorrhagic congestion (Fig 5).

\section{DISCUSSION}

In the present study, we used a bipolar RFA system in an attempt to produce an ablation zone with a minor-axis diameter greater than the distance from the probe tip to the posterior vertebral body cortex, but caused no histologic evidence of spinal cord injury due to the insulating effect of the posterior cortex. Previous animal experiments assessing the safety of RFA in close proximity to the spinal cord have been inconclusive. Dupuy et $\mathrm{al}^{12}$ safely performed monopolar RFA of the posterior vertebral body for 10 minutes, but the maximum temperature generated within the bone was only $48^{\circ} \mathrm{C}$, which is below the cytotoxic threshold. In a subsequent porcine study, monopolar RFA performed flush with the posterior vertebral body cortex for 10 minutes at a constant temperature of $90^{\circ} \mathrm{C}$ at the probe tip caused paraplegia with histologically confirmed spinal cord injury. ${ }^{13}$ However, this latter experiment does not reflect clinical practice because adequate ablation of tumor within the posterior vertebral body only requires that the periphery of the ablation zone reach $60^{\circ} \mathrm{C}$ for a matter of seconds. ${ }^{1}$

In contrast with these previous studies, the ablation system used in the present experiment has several features that facilitate its safe use in the spine. First, the distal segment of the probe articulates up to $90^{\circ}$, enabling accurate placement of the tip with respect to the posterior vertebral body cortex. Second, the 2 active thermocouples incorporated into the shaft of the probe allow real-time estimation of the dimensions of the ablation zone. Last, monopolar systems require placement of a skin-grounding pad, which, if placed incorrectly, can cause a skin burn. This risk is eliminated with the use of a bipolar probe. ${ }^{16}$ The RFA zones measured on gross pathology were smaller than those predicted from the manufacturer's preclinical data derived from a soft-tissue model. ${ }^{14}$ Potential reasons for this discrepancy include the higher impedance and lower thermal conductivity of bone compared with soft tissue and a heat sink effect from blood flow in the vertebrae and epidural space, as well as circulating CSF. ${ }^{1,12}$

In contrast to RFA, when we produced a cryoablation zone with a diameter greater than the distance from the probe tip to the posterior vertebral body cortex, histologic evaluation of the spinal cord at the treated level showed white matter necrosis with cavitation and axonal swelling and degeneration consistent with ablation-induced injury. Although there are no prior studies assessing the safety of cryoablation within the posterior vertebral body, this result is expected because increased bone density does not limit the propagation of the cryoablation zone. ${ }^{17}$ Several measures may be taken to minimize the risk of this complication clinically. First, the distance between the probe tip and the spinal 

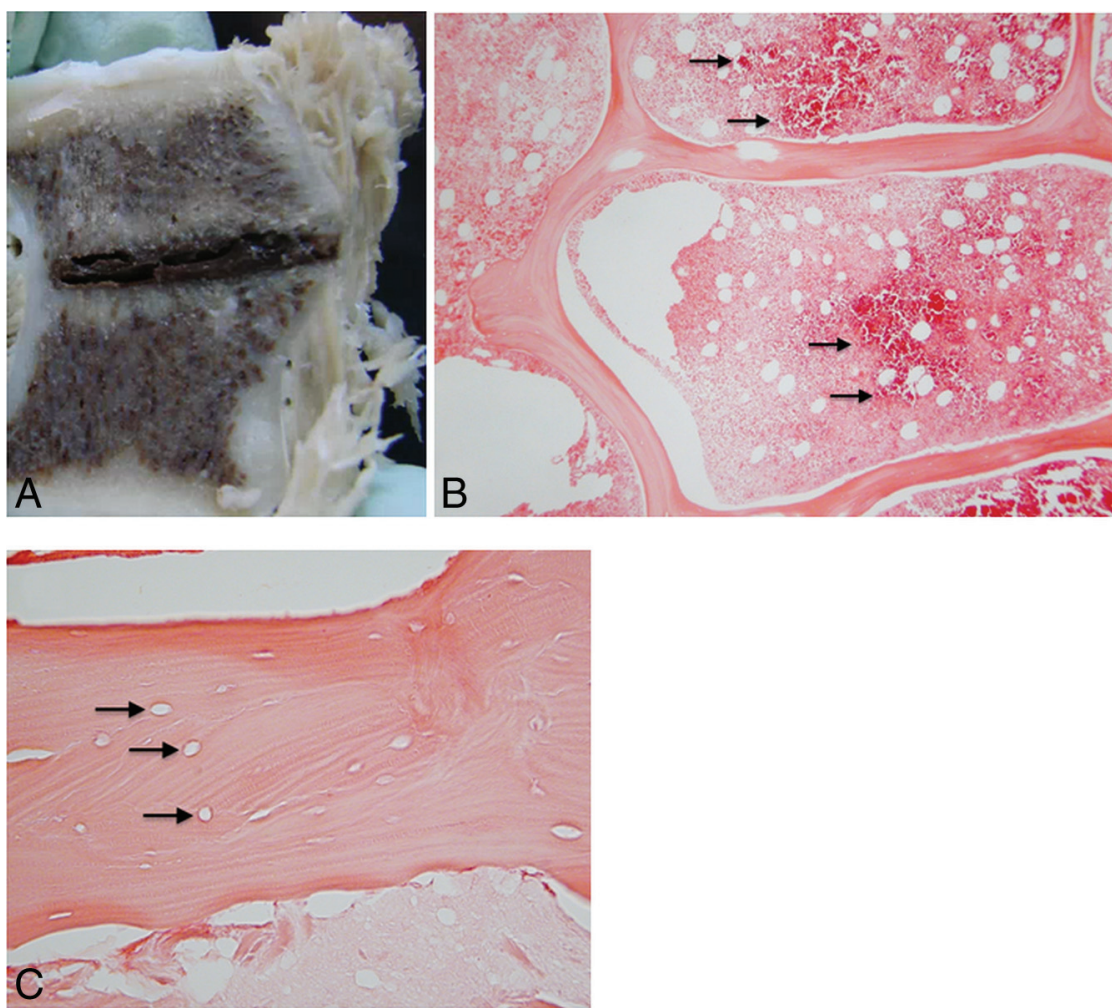

FIG 5. A, Gross pathology of the vertebral body shown in Fig 2 cut along the plane of the radiofrequency ablation probe tract. The tract is filled with hemorrhagic debris and is surrounded by a pale, tan zone of necrosis. $B$, Hematoxylin-eosin staining of the margin of the ablation zone at $\times 40$ total magnification shows a dense band of red blood cells (black arrows), which demarcate necrotic marrow on the left from the intact region on the right. C, At $\times 200$ magnification, the ablation zone shows empty lacunae (black arrows), representing a loss of osteocytes, within an intact trabecula.

canal must be accurately measured, and the cryoprobe and intensity and duration of the freeze cycles can then be chosen to ensure that the ablation zone does not exceed this distance. The dimensions of the cryoablation zones measured on gross pathology were within $1 \mathrm{~mm}$ of those predicted from the manufacturer's preclinical data derived from a $37^{\circ} \mathrm{C}$ gel bath model. Second, a coaxial needle with an inner thermocouple can be placed through the neural foramen to monitor the temperature in the epidural space, while the outer cannula is used to inject carbon dioxide for neural insulation and room temperature 5\% dextrose in water to warm the epidural space. ${ }^{18}$ Last, the use of motorevoked potential monitoring and peripheral motor nerve electrostimulation to prevent iatrogenic cryoablation nerve injury have been described. ${ }^{19,20}$

The present experiment also showed that the posterior vertebral body cortex is not a barrier to MWA because this ablation zone also extended into the spinal canal and caused histologically evident spinal cord injury. Like cryoablation, this result is expected because MWA is also non-impedance-based. However, the efficiency with which tissue absorbs microwave energy is proportional to its water content, and soft tissue is $>70 \%$ water compared with the approximately $20 \%$ water content of bone. ${ }^{21,22}$ Therefore, one would expect the MWA zones in the spine to be smaller than those expected from preclinical data derived from an ex vivo soft-tissue model. Yet, such a discrepancy was not observed. Accurate sizing of the MWA zone to remain confined to the vertebral body and injection of carbon dioxide and room temperature $5 \%$ dextrose in water into the epidural space may increase the safety of the procedure.

The ablation-induced marrow changes on MR imaging, gross pathology, and histopathology were consistent across all 3 modalities. The necrotic ablation zone was depicted on postcontrast T1weighted MR imaging with fat suppression as a nonenhancing area surrounded by a thin, enhancing rim of granulation tissue. T2 hyperintense enhancing residual tumor can appear similar to the rim of the ablation cavity. In some cases, FDG PET/CT or biopsy may be needed to differentiate residual tumor from postablation change. ${ }^{15}$ The ablation zone dimensions of all 3 modalities measured on gross pathology were slightly larger than those measured on MR imaging. In the absence of a plausible physiologic explanation, the discrepancy is likely related to the resolution limitations of MR imaging.

A limitation of this study is that the correlations between technical ablation parameters and ablation zone dimensions were derived in normal bone, and these correlations may be altered by the presence of tumor. Also, the derived correlations are specific to the ablation systems used in this experiment and cannot be extrapolated to other devices.

\section{CONCLUSIONS}

Intact posterior vertebral body cortex in normal sheep may protect the spinal cord from radiofrequency ablation-induced injury, but not against microwave or cryoablation injury. Ablation dimensions produced by microwave and cryoablation are similar to those expected, while RFA volumes are smaller. Postablation marrow changes in normal spinal marrow include a rim of enhancement at the margin of the ablation zone.

Disclosures: Adam N. Wallace—RELATED: Grant: Medtronic, Galil, DFINE, Comments: received donated ablation probes from these companies.* Travis J. Hillen-UNRELATED: Consultancy: Merit Medical, Comments: honorarium for proctoring physician education courses. Jack W. Jennings-UNRELATED: Consultancy: Galil, Medtronic, Merit Medical, Bard Medical; Payment for Lectures Including Service on Speakers Bureaus: Merit Medical and Medtronic Speaker Panels; Travel/Accommodations/Meeting Expenses Unrelated to Activities Listed: Comments: for meetings in which Speaker Panel duties were performed. *Money paid to the institution.

\section{REFERENCES}

1. Brace CL. Radiofrequency and microwave ablation of the liver, lung, kidney, and bone: what are the differences? Curr Probl Diagn Radiol 2009;38:135-43 CrossRef Medline

2. Rybak LD. Fire and ice: thermal ablation of musculoskeletal tumors. Radiol Clin North Am 2009;47:455-69 CrossRef Medline 
3. Wallace AN, Greenwood TJ, Jennings JW. Radiofrequency ablation and vertebral augmentation for palliation of painful spinal metastases. J Neurooncol 2015;124:111-18 CrossRef Medline

4. Anchala PR, Irving WD, Hillen TJ, et al. Treatment of metastatic spinal lesions with a navigational bipolar radiofrequency ablation device: a multicenter retrospective study. Pain Physician 2014;17: 317-27 Medline

5. Tomasian A, Wallace A, Northrup B, et al. Spine cryoablation: pain palliation and local tumor control for vertebral metastases. AJNR Am J Neuroradiol 2016;37:189-95 CrossRef Medline

6. Kastler A, Alnassan H, Aubry S, et al. Microwave thermal ablation of spinal metastatic bone tumors. J Vasc Interv Radiol 2014;25:1470-75 CrossRef Medline

7. Pusceddu C, Sotgia B, Fele RM, et al. Combined microwave ablation and cementoplasty in patients with painful bone metastases at high risk of fracture. Cardiovasc Intervent Radiol 2016;39:74-80 CrossRef Medline

8. Wallace AN, Tomasian A, Vaswani D, et al. Radiographic local control of spinal metastases with percutaneous radiofrequency ablation and vertebral augmentation. AJNR Am J Neuroradiol 2016;37: 759-65 CrossRef Medline

9. Wallace AN, Robinson CG, Meyer J, et al. The metastatic spine disease multidisciplinary working group algorithms. Oncologist 2015; 20:1205-15 CrossRef Medline

10. Algra PR, Heimans JJ, Valk J, et al. Do metastases in vertebrae begin in the body or the pedicles? Imaging study in 45 patients. AJR Am J Roentgenol 1992;158:1275-79 CrossRef Medline

11. Klimo P Jr, Thompson CJ, Kestle JR, et al. A meta-analysis of surgery versus conventional radiotherapy for the treatment of metastatic spinal epidural disease. Neuro Oncol 2005;7:64-76 CrossRef Medline

12. Dupuy DE, Hong R, Oliver B, et al. Radiofrequency ablation of spi- nal tumors: temperature distribution in the spinal canal. $A J R A m J$ Roentgenol 2000;175:1263-66 CrossRef Medline

13. Nour SG, Aschoff AJ, Mitchell IC, et al. MR imaging-guided radiofrequency thermal ablation of the lumbar vertebrae in porcine models. Radiology 2002;224:452-62 CrossRef Medline

14. Singh S, Saha S. Electrical properties of bone: a review. Clinl Orthop Relat Res 1984;186:249-71

15. Wallace AN, Greenwood TJ, Jennings JW. Use of imaging in the management of metastatic spine disease with percutaneous ablation and vertebral augmentation. AJR Am J Roentgenol 2015;205: 434-41 CrossRef Medline

16. Wallace AN, Tomasian A, Chang RO, et al. Treatment of osteoid osteomas using a navigational bipolar radiofrequency ablation system. Cardiovasc Intervent Radiol 2016;39:768-72 CrossRef Medline

17. Gangi A, Buy X. Percutaneous bone tumor management. Semin Intervent Radiol 2010;27:124-36 CrossRef Medline

18. Buy X, Tok CH, Szwarc D, et al. Thermal protection during percutaneous thermal ablation procedures: interest of carbon dioxide dissection and temperature monitoring. Cardiovasc Intervent Radiol 2009;32:529-34 CrossRef Medline

19. Kurup AN, Morris JM, Boon AJ, et al. Motor evoked potential monitoring during cryoablation of musculoskeletal tumors. J Vasc Interv Radiol 2014;25:1657-64 CrossRef Medline

20. Tsoumakidou G, Garnon J, Ramamurthy N, et al. Interest of electrostimulation of peripheral motor nerves during percutaneous thermal ablation. Cardiovasc Intervent Radiol 2013;36:1624-28 CrossRef Medline

21. Emery JL, Finch E. The fat and water content of the left and right liver before and after birth. Arch Dis Child 1954;29:242-47 Medline

22. Smith JW. Observations on the water content of bone. J Bone Joint Surg Br 1964;46:553-62 Medline 